\title{
Noninvasive imaging of pancreatic inflammation and its reversal in type 1 diabetes
}

\author{
Stuart E. Turvey, ${ }^{1}$ Eric Swart, ${ }^{2}$ Maria C. Denis, ${ }^{1}$ Umar Mahmood, ${ }^{2}$ \\ Christophe Benoist, ${ }^{1}$ Ralph Weissleder, ${ }^{2}$ and Diane Mathis ${ }^{1}$ \\ ${ }^{1}$ Section on Immunology and Immunogenetics, Joslin Diabetes Center, and Department of Medicine, \\ Brigham and Women's Hospital, Harvard Medical School, Boston, Massachusetts, USA. ${ }^{2}$ Center for Molecular Imaging Research, \\ Massachusetts General Hospital, and Harvard Medical School, Boston, Massachusetts, USA.
}

\begin{abstract}
A major stumbling block for research on and treatment of type 1 diabetes is the inability to directly, but noninvasively, visualize the lymphocytic/inflammatory lesions in the pancreatic islets. One potential approach to surmounting this impediment is to exploit MRI of magnetic nanoparticles (MNP) to visualize changes in the microvasculature that invariably accompany inflammation. MNP-MRI did indeed detect vascular leakage in association with insulitis in murine models of type 1 diabetes, permitting noninvasive visualization of the inflammatory lesions in vivo in real time. We demonstrate, in proof-of-principle experiments, that this strategy allows one to predict, within 3 days of completing treatment with an anti-CD3 monoclonal antibody, which NOD mice with recent-onset diabetes are responding to therapy and may eventually be cured. Importantly, an essentially identical MNP-MRI strategy has previously been used with great success to image lymph node metastases in prostate cancer patients. This success strongly argues for rapid translation of these preclinical observations to prediction and/or stratification of type 1 diabetes and treatment of individuals with the disease; this would provide a crucially needed early predictor of response to therapy.
\end{abstract}

\section{Introduction}

Type 1 diabetes is an autoimmune disease characterized by lymphocytic infiltration of the pancreatic islets, culminating in specific destruction of insulin-producing $\beta$ cells (1-3). This immunological process unfolds over a variable number of years, resulting in clinically detectable hyperglycemia and, ultimately, diagnosis of diabetes. At the present time, the islet infiltrate, termed insulitis, is only detectable by biopsy (4), usually at autopsy.

The ability to detect lymphocytic infiltration and associated inflammation of the pancreas through noninvasive means would most likely have clinical benefits in 2 major areas. The first is diagnostic: helping physicians distinguish patients that have an atypical type 1 rather than type 2 diabetes or identifying individuals with preclinical type 1 diabetes as early as possible, ensuring early initiation of therapy and regular follow-up. The second area, of perhaps even greater potential, involves following patients who are undergoing interventions to prevent or reverse overt disease. Currently, the only accepted endpoint for trials in these areas is the clinical diagnosis of diabetes. This makes the trials lengthy and expensive - for example, 7 years for the parenteral insulin treatment arm of the Diabetes Prevention Trial-Type 1 (5). An accurate method for noninvasively following the progression or regression of insulitis might permit the early recognition and monitoring of potentially beneficial therapies and the discarding of ineffective treatments.

The complex cellular infiltrate associated with autoimmune diabetes is accompanied by a range of alterations in the micro-

Nonstandard abbreviations used: CPA, cyclophosphamide; MNP, magnetic nanoparticles; ROI, region of interest; VVF, vascular volume fraction.

Conflict of interest: The authors have declared that no conflict of interest exists.

Citation for this article: J. Clin. Invest. 115:2454-2461 (2005).

doi:10.1172/JCI25048. vasculature, including modification of endothelial cells, vascular swelling, increased islet blood flow, and edema (6-13). These microvascular changes may be amenable to visualization with a range of newly described imaging probes and techniques (14). A particularly attractive approach is the use of high spatial resolution MRI combined with magnetic nanoparticles (MNP) in deriving physiologic (15) and molecular (16) information. One highly useful MNP platform has been the application of long-circulating, dextran-coated, monocrystalline, superparamagnetic iron oxides that exhibit strong magnetic behavior detectable by high-resolution MRI. The attractiveness of this approach is heightened by our recent experiments demonstrating that it can be used to identify insulitis in the BDC2.5 TCR Tg mouse model of type 1 diabetes (17) coupled with its demonstrated safety and utility in the visualization of small and otherwise undetectable lymph node metastases in patients with prostate cancer (18).

Our earlier report (17) began with and focused on ex vivo confocal microscopic analysis of the accumulation of fluorescently labeled nanoparticles in the pancreas, in order to establish some of the basic parameters of the approach. It demonstrated that vascular leakage occurs in association with insulitis in the exaggerated BDC2.5 model, that this leakage can be detected as an accumulation of labeled nanoparticles in invading macrophages, and that nanoparticle accumulation is well correlated with the aggressivity of the islet lesion. Similar though decidedly less striking results were obtained using the same ex vivo imaging modality in the more complex, and thus probably more relevant, NOD mouse model. In a final experiment, we demonstrated the ability to identify infiltrated BDC2.5 islets in vivo using MNP-MRI. Here we build on these preliminary observations to exploit this noninvasive in vivo imaging strategy to track pancreatic inflammation during the unfolding of autoimmune diabetes in BDC2.5 and then in NOD 


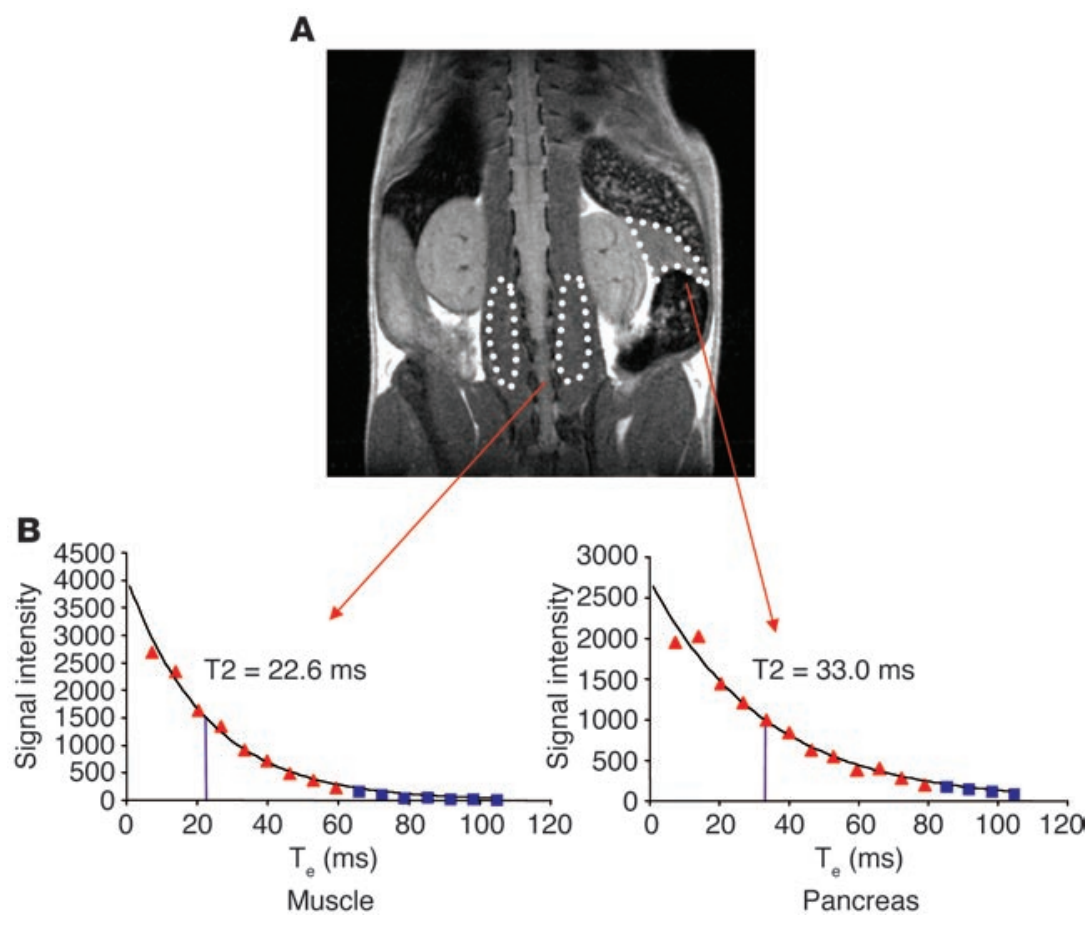

Figure 1

Mapping organ-specific T2 values. Multiple-slice, multiple-echo T2-weighted spin-echo sequences were acquired, and ROIs for analysis were defined manually on the pancreas or paraspinal muscles. (A) Coronal view of a mouse visualized by MRI. Pancreas and paraspinal muscle ROls are outlined with dotted lines. (B) T2 values for the individual tissues outlined in $\mathbf{A}$ were calculated by fitting a standard exponential relaxation model (Signal intensity $=B_{0} \cdot e^{-\left(T_{e} / T 2\right)}$; where $B_{0}$ is initial magnetic field strength $[1.5 \mathrm{~T}], T_{e}$ is echo time, and T2 is T2 relaxation time) to the data within the ROIs. Values shown as blue squares were below background and were not included in analysis.

mice. Encouraging results from these experiments prompted us to apply the technique to 2 clinically relevant issues: predicting an individual's risk of developing autoimmune diabetes and early monitoring of the effectiveness of an immunomodulatory therapy aimed at reversing diabetes.

\section{Results}

Seeing the evolution of pancreatic inflammation during the development of cyclophosphamide-induced diabetes. The overarching goal of these experiments was to provide proof-of-principle preclinical data on mouse models of type 1 diabetes to guide us in safely and successfully applying an in vivo MNP-MRI technique to patients who have or are at risk of autoimmune diabetes. A logical first step was to track the inflammatory signals arising from the pancreas during disease unfolding. As it is impossible to predict the exact age at which diabetes will spontaneously appear in a given NOD mouse, we initially employed the simpler BDC2.5 TCR Tg model. BDC2.5/NOD mice carry the rearranged TCR genes from a diabetogenic $\mathrm{CD}^{+} \mathrm{T}$ cell clone, isolated from a female NOD mouse, that recognizes an islet $-\beta$ cell antigen presented by the MHC class II molecule $\mathrm{Ag}^{\mathrm{g}}$ (19-21). These mice have a $\mathrm{T}$ cell repertoire highly skewed toward $\beta$ cell reactivity. All animals exhibit insulitis abruptly between 2 and 3 weeks of age, but the rate and penetrance of diabetes development varies according to the genetic background, a phenomenon now known to reflect immunoregulation (22). Treatment of BDC2.5/NOD mice with cyclophosphamide (CPA) destabilizes the immunoregulatory balance and induces autoimmune diabetes in $100 \%$ of animals between 4 and 10 days after injection (23). Such rapid, highly reproducible kinetics allow a detailed temporal analysis of inflammatory changes occurring within the pancreas during the development of autoimmune diabetes.

In order to synchronize development of diabetes, 6- to 8-week-old female BDC2.5/NOD mice were injected with CPA, and MRI was performed at various time points thereafter. Routinely, animals were anesthetized and a baseline MRI obtained. Without removing the mice from the MRI scanner, we injected MNP intravenously, and a second image was obtained immediately in order to yield vascular volume data from the blood-borne nanoparticles. Mice underwent a third scan 24 hours later to provide information about microvascular permeability. A region of interest (ROI) was drawn around the pancreas (Figure 1), and the $\mathrm{T} 2$ value of the organ was calculated, with the presence of nanoparticles within an organ decreasing its $\mathrm{T} 2$ value.

Little difference was detected in the pancreatic $\mathrm{T} 2$ values of control animals and those imaged on day 1 or 2 after treatment with CPA (Figure 2A). In both cases, the $\mathrm{T} 2$ response was as expected in the absence of pancreatic inflammation. It dropped below the baseline value immediately after MNP injection due to the presence of circulating iron oxide within the bloodstream but recovered to baseline within 24 hours, reflecting an absence of nanoparticle leakage across the intact endothelial barrier. In contrast, striking alterations in the pancreatic T2 values were apparent on days 3 or 4 and 5 or 6 after CPA treatment (plotted in Figure 2A; shown in Figure 3). On these days, the values before and immediately after MNP injection had increased markedly. This augmentation probably reflects the fact that baseline T2 is sensitive to alterations in free water, the increase in this instance probably signifying edema within the organ. The $\mathrm{T} 2$ value on these days had substantially dropped by 24 hours after MNP injection, indicating that the probe had extravasated from the inflamed pancreatic microvasculature. The resulting accumulation of iron oxide within the pancreas, most likely within macrophages, altered its local magnetic characteristics and thereby decreased its total T2 value to below that seen on most other days studied, despite the effects of increased $\mathrm{T} 2$ from edema at these time points.

It was striking how well the MRI observations, made noninvasively on live animals, reflected the histological changes that accompanied CPA-triggered diabetes in the BDC2.5/NOD model (23). Little morphological change was detectable on days 1 and 2 after injection of CPA (data not shown). On day 6 , most of the islets had lost their characteristic structure due to massive lymphocytic infiltration, and this disintegration was accompanied by edema and separation of the surrounding acinar lobules (Figure 3).

We went on to scan mice at 2 time points late after CPA treatment, when $\beta$ cell destruction was complete and the local immune response was resolving (Figure 2A). On day 14 or 15, the 

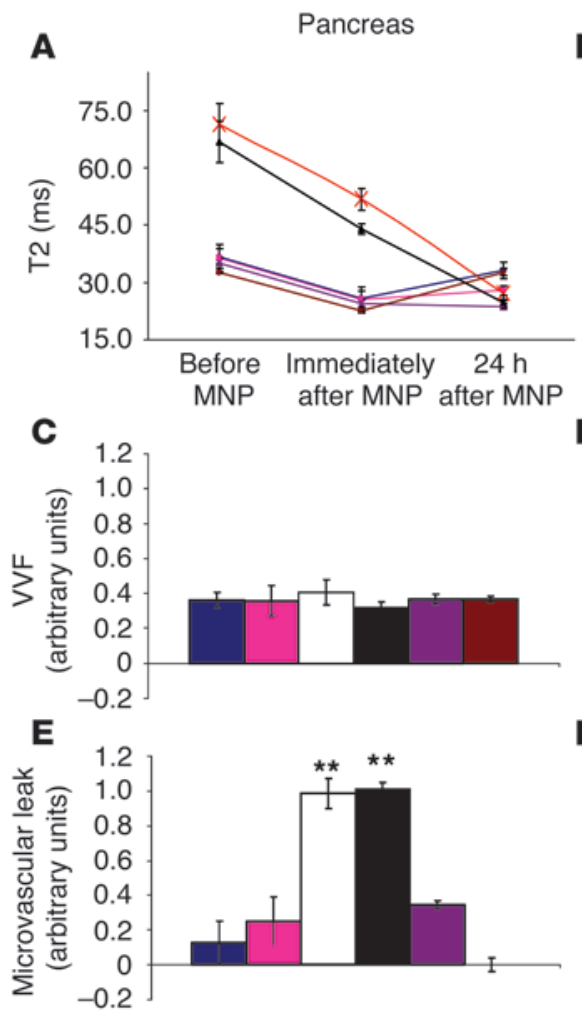

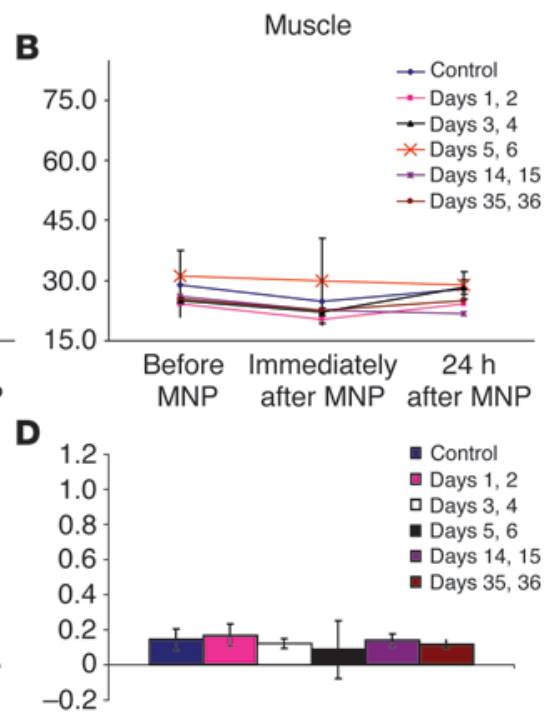

$\mathbf{F}$

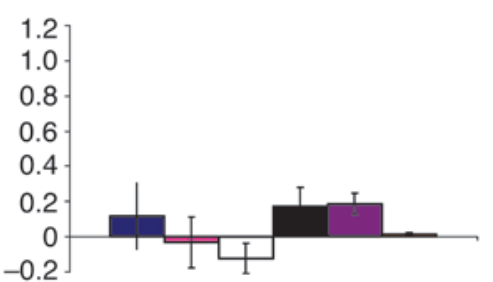

Figure 2

Tracking pancreatic inflammation during the development of autoimmune diabetes. Young female BDC2.5/NOD mice were injected with CPA to provoke autoimmune diabetes and were imaged at the indicated time points. Each group contained 4-7 mice. T2 values for pancreas (A) and paraspinal muscles (B) are shown. VVF (C and D) and microvascular leak ( $\mathbf{E}$ and $\mathbf{F}$ ) were estimated for each organ using formulae described in Methods. ${ }^{\star \star} P<0.01$.

pancreatic T2 values at baseline and immediately following MNP injection were indistinguishable from those of controls, suggesting that the edema had resolved. However, the T2 values at 24 hours after MNP injection remained below baseline, indicating that pancreatic inflammation continued to enhance microvascular leak in these animals. On day 35 or 36 after CPA treatment, pancreatic $\mathrm{T} 2$ values had completely returned to the control level, signifying that at this late stage after islet destruction, microvascular integrity had returned to the normal, noninflamed state. Importantly, the inflammatory changes were specific to the pancreas, as no alterations in $\mathrm{T} 2$ values were observed in other tissues, such as muscle (Figure 2B).

Inflammation can be accompanied by a range of microvascular changes, including transient vasoconstriction, vasodilatation, increased blood flow, and vascular leakage (24). One of the attributes of MNP-MRI is the ability to precisely quantify the relative contribution of 2 important microvascular parameters. First, this imaging strategy can measure alterations in vascular volume fraction (VVF) of the pancreas, caused by acute vasodilation or vasoconstriction, by comparing the baseline $\mathrm{T} 2$ value with the $\mathrm{T} 2$ value obtained immediately after MNP injection (15). Second, comparison of the baseline $\mathrm{T} 2$ value with the $\mathrm{T} 2$ value measured 24 hours after MNP injection yields information about vascular leakiness. The VVF of the pancreas remained unchanged during the evolution of CPA-induced diabetes in BDC2.5/NOD mice
(Figure 2C). In contrast, pancreatic microvascular leak followed a crescendo-decrescendo pattern, peaking at the time of maximal islet destruction (Figure 2E). No significant alterations in either VVF or microvascular leak were observed in the muscle (Figure 3, D and F). Thus, this MNP-MRI approach allows one to infer, noninvasively from live animals, the major morphological features that accompany the development of autoimmune diabetes triggered by CPA in BDC2.5/NOD mice.

Alterations in pancreatic inflammation associated with spontaneous autoimmune diabetes. Given our interest in eventually imaging the development of autoimmune diabetes in humans, we turned next to the more complex, and therefore more clinically relevant, NOD mouse model. The critical features of human type 1 diabetes, especially its genetic and immunological aspects, are very similar to those of the NOD disease, making this strain currently the most popular model for studying autoimmune diabetes. Since the $\mathrm{T}$ cell repertoire in standard NOD mice is much less skewed toward $\beta$ cell reactivity than in the engineered BDC2.5/NOD model, we expected that the inflammatory signal during the spontaneous development of autoimmune diabetes in NOD animals would be more subtle.

We were primarily interested in answering 2 questions: Do mice recently diagnosed with spontaneous autoimmune diabetes have more pancreatic inflammation than nondiabetic animals? Is it possible to differentiate new-onset diabetic animals from those at risk of developing autoimmune diabetes based on noninvasive measures of pancreatic microvascular leakiness? Three groups of mice were compared in order to address these questions. Female NOD mice with new-onset diabetes $(n=9)$, defined as hyperglycemia of less than 7 days duration, were compared with age-matched, nondiabetic female littermates $(n=7)$. E $\alpha 16 / \mathrm{NOD}$ mice $(n=13)$ served as a negative control - these animals have seemingly normal $\mathrm{T}$ and B lymphocyte compartments but are completely protected from insulitis and the subsequent development of autoimmune diabetes through transgene-induced expression of the $\mathrm{E}$ complex on all cells normally displaying MHC class II molecules (25).

The pancreatic $\mathrm{T} 2$ values obtained at baseline and immediately after MNP injection were indistinguishable among the 3 groups (Figure 4A). These similarities in NOD mice contrast with the alterations seen in the BDC2.5/NOD animals, indicating that pronounced pancreatic edema is not a general feature of spontaneous autoimmune diabetes but is rather a particularity of the exaggerated TCR Tg model. Interesting differences became apparent only 24 hours after MNP administration, which demonstrated that diabetes in NOD mice is also associated with alterations in microvascular leakiness rather than in pancreatic VVF caused by vasodilation or vasoconstriction. T2 values in the noninsulitic Ea16/NOD mice (Figure 4B) recovered to baseline 24 hours after MNP injection, indicating that there was no significant vascu- 


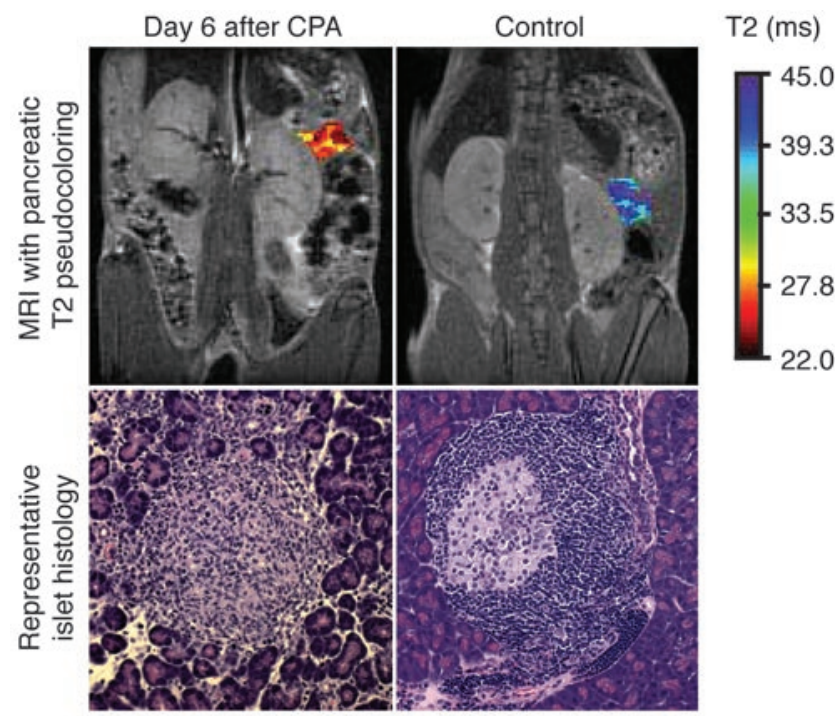

Figure 3

Severity of insulitis revealed by noninvasive MNP-MRI. Young female BDC2.5/NOD mice were imaged on day 6 after CPA treatment and 24 hours after MNP injection. A pseudocolor was assigned to the pancreas, reflecting the T2 value of the organ. Photomicrographs show representative islet histology from these animals. Magnification, $\times 20$.

lar leak in the absence of insulitis. In contrast, the T2 in heavily infiltrated new-onset diabetic animals (Figure 4C) remained well below the baseline value and was significantly lower (and hence vascular leak was higher) than in both the noninsulitic Ea16/ NOD group $(P=0.00004)$ and the nondiabetic NOD littermates $(P=0.01)$. The latter mice, which had insulitis of varying severity, there being significant individual-to-individual variation in disease progression in the standard NOD model, had T2 levels that

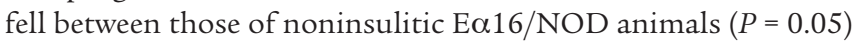
and the new-onset diabetic animals $(P=0.01)$. Thus, the evolution of pancreatic inflammation during the development of spontaneous diabetes in NOD mice can be monitored noninvasively in living animals using MNP-MRI.

Will noninvasive MRI predict the eventual development of diabetes? Extensive effort has focused on identifying markers that signal the imminent conversion of preclinical insulitis to clinical diabetes. To date, for both the NOD mouse model and human patients, the serum titers of autoantibodies directed against a defined set of islet-cell antigens have proven to be the most reliable indicators, as highlighted by recent results from the Diabetes Prevention Trial-Type $1(5,26)$. However, autoantibodies are at best an indirect measure of pancreas inflammation, and a noninvasive means of directly following diabetes progression in vivo would have a number of important applications.

Therefore, a cohort of nondiabetic female NOD mice was scanned at 13 and 20 weeks of age, and the group was followed for the spontaneous development of diabetes up until 30 weeks. Unfortunately, it seems that this imaging approach is unlikely to be useful for prediction of diabetes risk, at least in the long-term time frame tested here (Figure 5). Data from the scan performed at 13 weeks showed no correlation between the pancreatic T2 value 24 hours after MNP injection and the time at which diabetes was eventually diagnosed (Figure 5A). However, some inter- esting insights were gained from the 20-week scans (Figure 5B). The 3 mice with the lowest pancreatic T2 values (reflecting the highest microvascular leak) recorded at the 20 -week scan all became diabetic within 1 week of the scan (Figure 5B, circle). In contrast, mice that became diabetic at later times had T2 values essentially indistinguishable from those of the group that remained normoglycemic beyond 30 weeks. Together, these data suggest that noninvasive monitoring of microvascular leakage provides important information concerning acute inflammation of the pancreas, i.e., that associated with recent-onset or impending diabetes. However, this particular imaging strategy is unlikely to be helpful in assessing an individual's long-term risk of developing autoimmune diabetes.

Monitoring alterations in pancreatic inflammation following anti-CD3 monoclonal antibody treatment. A major potential application of this imaging technique is in monitoring acute changes in pancreatic inflammation in patients undergoing trial interventions to treat or prevent type 1 diabetes. The long half-life of islet autoantibodies renders them unresponsive to acute changes in autoimmune attack of the pancreas. In contrast, microvascular permeability is likely to change rapidly with successful immunointervention.

Short-term treatment of NOD mice with mAbs directed against the $\mathrm{CD} 3 \varepsilon$ subunit of the TCR reverses recent-onset diabetes and restores self tolerance to $\beta$ cell antigens in a variable proportion of animals (27-29). Mice responding to this treatment generally return to normoglycemia within 2 to 4 weeks. The rate of diabetes remission varies with disease severity and is influenced by multiple factors, including the breeding facilities

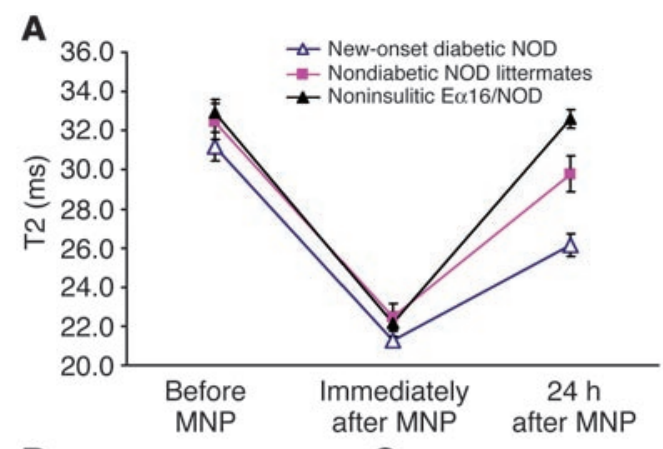

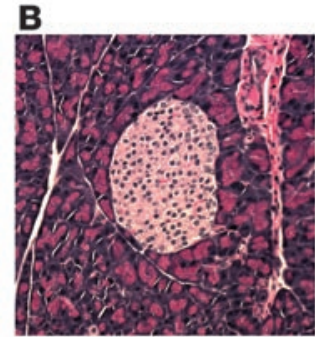

$\mathrm{E} \alpha 16 / \mathrm{NOD}$
C

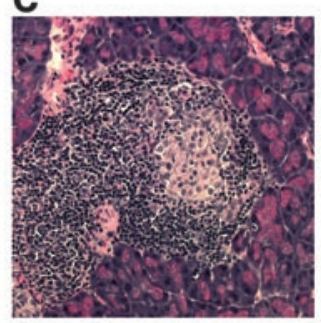

New-onset diabetic

\section{Figure 4}

Detection of pancreatic inflammation associated with spontaneous autoimmune diabetes. Female NOD mice with new-onset diabetes of less than 7 days' duration $(n=9)$, nondiabetic female littermates of the diabetic animals $(n=7)$, and age-matched noninsulitic E $\alpha 16 / \mathrm{NOD}$ mice $(n=13)$ underwent MRI imaging according to the protocol described in Methods. (A) Mean pancreatic T2 values \pm SEM for each group. (B and C) Representative islet histology from $\mathrm{E} \alpha 16 / \mathrm{NOD}$ and new-onset diabetic NOD animals. Magnification, $\times 20$. 

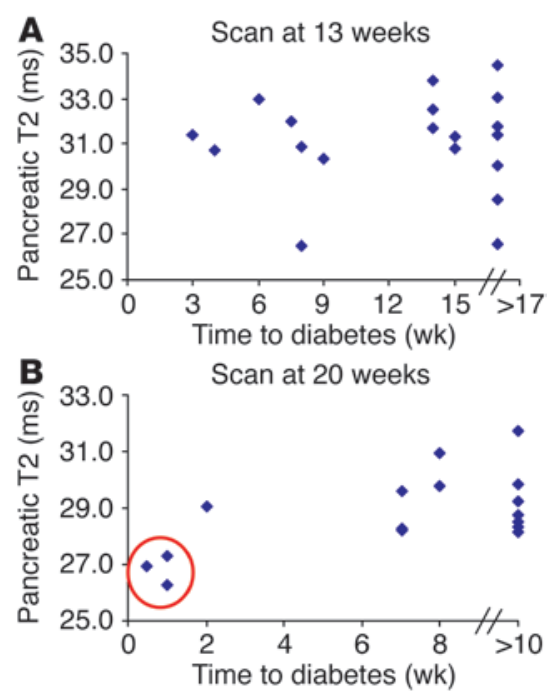

employed (30); our protocol generally results in a remission rate of $40-60 \%$. Imaging this therapeutic regime in mice is particularly attractive because an analogous approach has been applied clinically using a nonactivating humanized mAb against CD3 and was found to mitigate the deterioration in insulin production and to improve metabolic control in patients for up to 12 months following treatment (31).

To specifically monitor the phases previously shown to reflect the restoration of immunological self tolerance, we performed MRI analyses of mice on days 4, 8, and 18 following initiation of anti-CD3 mAb therapy (Figure 6A). On days 4-8, the pancreas in mice responding favorably to treatment should be cleared of infiltrate; on day 18, the lymphocytes will have reappeared, but will accumulate in a peri-islet pattern. At these early time points, it was often unclear clinically which animals had responded favorably to the immunointervention. Since some animals in this study remained profoundly hyperglycemic for longer than 3 weeks (i.e., the anti-CD3 nonresponder group imaged at day 18),

\section{Figure 6}

Noninvasive imaging of pancreatic inflammation can predict clinical response to anti-CD3 immunotherapy. Female NOD mice with very recent onset of diabetes were treated with either anti-CD3 or control $\mathrm{F}\left(\mathrm{ab} \mathrm{b}_{2}\right)_{2}$ fragments for 5 consecutive days as described in Methods. MRI was performed 24 hours after MNP injection on days 4,8 , and 18 after starting mAb immunotherapy. (A) Results from long-term responders rendered normoglycemic following $\mathrm{F}\left(a b^{\prime}\right)_{2}$ treatment (triangles) are compared with those of mice failing to respond to therapy, i.e., antiCD3 nonresponders (squares) and control antibody nonresponders (circles). Since some mice remained profoundly hyperglycemic for over 3 weeks in this experimental series, pancreas/muscle T2 ratio is presented to control for nonspecific alterations in MRI parameters induced by these global metabolic changes. Groups represent the following number of mice: day 4: responders, $n=4$, nonresponders, $n=6$; day 8: responders, $n=6$, nonresponders, $n=4$, control mAb treated, $n=3$; day 18: responders, $n=4$, nonresponders, $n=6$. (B) Pancreas/ muscle $\mathrm{T} 2$ ratio is plotted against the serum glucose measured at the time of scanning $(n=33)$, showing no correlation between pancreatic inflammation as determined by MNP-MRI and serum glucose.

\section{Figure 5}

Prediction of diabetes risk with noninvasive imaging. Nondiabetic female NOD mice underwent MRI scanning at 13 (A) and 20 (B) weeks according to the protocol described in Methods. Animals were followed for the spontaneous development of diabetes until 30 weeks. Correlation between T2 value of the pancreas obtained 24 hours after MNP injection and time to diagnosis of overt diabetes is shown. Circle in $\mathbf{B}$ indicates 3 mice with the lowest recorded pancreatic T2 values and shortest time to diabetes onset.

the pancreas/muscle T2 ratio was calculated in mice scanned 24 hours after MNP injection in order to control for any nonspecific changes in MRI parameters caused by these metabolic abnormalities. On day 4 of the 5-day treatment course, no difference was detected between animals that eventually responded to antiCD3 therapy by long-term restoration of normoglycemia and those that failed to respond (responders, $n=4$; nonresponders, $n=6)$. On day 8 after initiation of anti-CD3 therapy, mice with a favorable response had significantly less microvascular leak in the pancreas than did either the animals that did not respond to therapy $(P=0.01)$ or those treated with a control $\mathrm{mAb}(P=0.005$; responders, $n=6$; nonresponders, $n=4$; control $\mathrm{mAb}$-treated, $n=3)$. These differences were still detectable at day 18 although the magnitude of the differences was less $(P=0.02$; responders, $n=4$; nonresponders, $n=6$ ).

To address the possibility that pancreatic inflammation as measured by MNP-MRI merely correlated with glycemic control, we plotted the pancreas/muscle $\mathrm{T} 2$ ratio against serum glucose at the time of scanning for individual mice in this experimental series $(n=33)$. There was marked variation in pancreas/muscle $\mathrm{T} 2$ ratio at all levels of serum glucose (Figure $6 \mathrm{~B}$ ) and hence no relationship between the degree of pancreatic inflammation and the level of hyperglycemia.

Thus, MR-based imaging can be used to noninvasively monitor changes in pancreatic inflammation following treatment with $\mathrm{mAbs}$ targeting CD3. Moreover, MNP-MRI identified animals with a favorable response to therapy as early as 3 days after completion of the course of anti-CD3 treatment (i.e., on day 8).
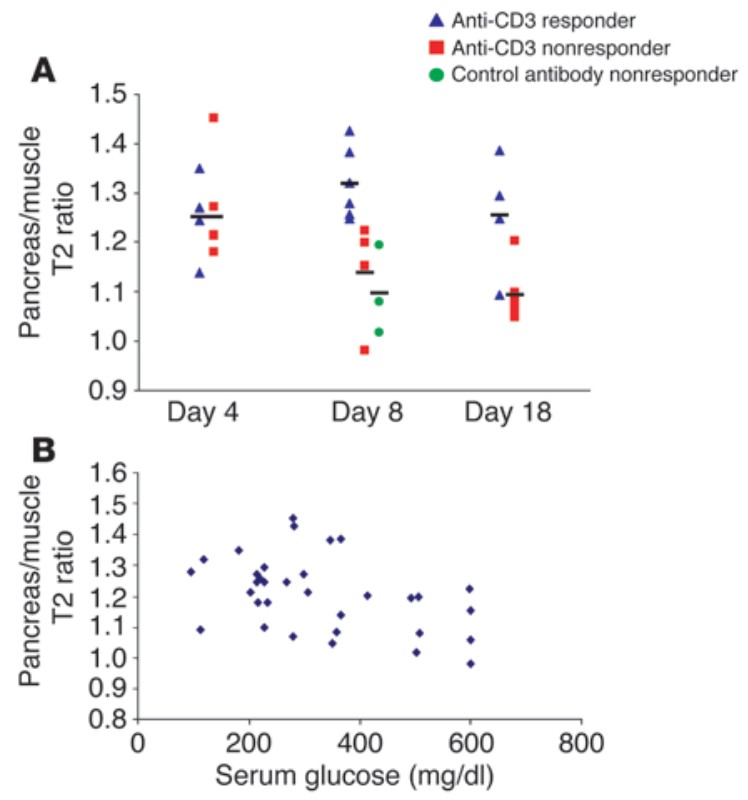


\section{Discussion}

Type 1 diabetes is an autoimmune disease characterized by lymphocytic infiltration of the pancreatic islets and, ultimately, destruction of insulin-producing $\beta$ cells. To date, no reliable method to directly but noninvasively detect and follow this lymphocyte invasion has been established. Due to the small size of the islets and the chronic nature of insulitis, conventional imaging methodologies - including computed tomography, nuclear magnetic resonance, and ultrasound techniques - have so far been unable to directly visualize insulitis (32).

Previous attempts to image pancreatic inflammation and subsequent $\beta$ cell destruction in autoimmune diabetes in vivo have used 2 distinct approaches. The first attempted to quantify residual $\beta$ cell mass in mice using a $\beta$ cell-specific mAb IC2 modified with a radioisotope chelator for nuclear imaging (33). Unfortunately, this system was limited by nonspecific accumulation of the probe in organs adjacent to the pancreas, such as the liver and spleen, preventing imaging in intact, living animals. The second approach, recently published, used MRI to track recruitment of diabetogenic CD8 ${ }^{+} \mathrm{T}$ cells to the pancreas (34). Since this method relied on antigen-specific labeling of autoreactive $T$ cells with a targeted MNP, it was entirely dependent upon knowledge of the islet antigen(s) recognized by autoreactive $\mathrm{T}$ cells - information that remains incomplete for mice and largely nonexistent for humans at this time (35-37).

We report the ability of a newly described imaging technique, specifically high-resolution MNP-MRI, to identify and quantify the vascular volume and permeability changes associated with inflammation of the pancreas during the development of autoimmune diabetes in the mouse. This method relies on measurement of the microvascular changes associated with inflammation and is not hampered by the variations in MHC alleles or autoreactive $\mathrm{T}$ cell specificities that limit other experimental approaches. Our enthusiasm for this technique is further heightened by the demonstrated safety and utility of an analogous protocol in the study of humans with prostate cancer (18).

When applied to preclinical mouse models of autoimmune diabetes, MNP-MRI allowed noninvasive, real-time quantification of pancreatic inflammation. In the simplified BDC2.5/ NOD model, microvascular changes developed dramatically 3 days after CPA injection, just 2 days prior to the onset of overt diabetes. Analysis of NOD mice, which more accurately model many of the features of human type 1 diabetes, confirmed the benefits of measuring nanoparticle-induced signal changes. This imaging strategy was able to differentiate between new-onset diabetics, nondiabetic NOD littermates at risk of developing diabetes, and noninsulitic E $\alpha 16 /$ NOD mice. These results suggest, by analogy, that MRI combined with MNP may be helpful in identifying human patients at immediate risk of developing autoimmune diabetes and in differentiating between those who have type 1 and type 2 diabetes.

The ability to monitor the response of an individual following an intervention designed to prevent or cure diabetes is an important clinical need that currently remains unmet. Investigators are left to rely on a range of imperfect parameters to monitor the response to any therapeutic intervention. Islet-cell antibodies (ICAs), with their long circulating half-life, are mute witnesses to acute changes in autoimmune islet damage $(38,39)$ Metabolic parameters, such as glycemic control, insulin requirements, and episodes of hypoglycemia, are highly variable and unreliable end points (40). Consequently, C-peptide, which is secreted by the pancreas on an equimolar basis with insulin, has emerged as the preferred measure of $\beta$ cell function (40). However, despite the established utility of measuring C-peptide levels following an immunointervention, they do not provide direct information about the pathology underlying type 1 diabetes - the autoimmune lesions within the pancreas. We predicted that changes in pancreatic inflammation, as measured by MNP-MRI, would provide a direct real-time readout of the effects of an immunointervention designed to reverse diabetes.

To test this hypothesis, we followed new-onset diabetic mice treated with mAbs specific for CD3E. Such treatment effectively reverses hyperglycemia in a proportion of animals and induces long-lasting protection from diabetes (27-29). It is of particular interest to focus on this therapeutic intervention because, based on the success of this therapy in rodent models, a humanized anti-CD3 mAb with decreased $\mathrm{Fc}$ receptor affinity is currently undergoing phase I/II trials in patients with recent-onset type 1 diabetes, and initial results have been promising (31). The tolerogenic effects of treating mice with anti-CD3 mAbs seem to evolve in 2 distinct phases (41): the first, occurring up to 7-8 days following initiation of antiCD3 therapy, entails clearing the infiltrating cells from the islets; the second, long-lasting phase is associated with active immunoregulation and the reappearance of a lymphocytic infiltrate that is now nondestructive and confined to the periphery of the islets. Noninvasive imaging in vivo allowed us to identify as early as 3 days after completing the anti-CD3 treatment course (i.e., on day 8) those animals benefiting from immunomodulation. Mice that responded favorably generally experienced complete normalization of blood glucose levels within 2 to 4 weeks following treatment; hence, we were able to detect the beneficial effects before it was clear which animals were destined to become normoglycemic. Interestingly, imaging on day 18 of the treatment protocol, a time when the regulated lymphocytic infiltrate is reappearing at the periphery of the islets, revealed a less marked difference in microvascular leak between mice responding favorably and those not responding to anti-CD3 therapy. The evolution of the T2 values may reflect a slight increase in microvascular leak associated with the accumulation of the immunoregulatory infiltrate in responding animals. Interestingly, a similar transient increase in microvascular leak was observed in BDC2.5/NOD mice during the development of a regulated lymphocytic islet infiltrate (17). While these results are quite striking and augur well for eventual translation to human anti$\mathrm{CD} 3 \mathrm{mAb}$ clinical trials, divergences between human and murine systems will need to be accounted for, including any mechanistic differences due to the different $m A$ bs employed.

We have developed a noninvasive method for monitoring autoimmune inflammation in the pancreas of a living animal. Using this imaging approach, we were able to track the insulitis that accompanies the development of autoimmune diabetes in induced and spontaneous mouse models and to follow the resolution of pancreatic inflammation after successful reversal of diabetes with anti-CD3 mAb therapy. We anticipate that these preclinical results will be useful in guiding the successful translation of this imaging technique to the care of patients with type 1 diabetes.

\section{Methods}

Mice. NOD/Lt, E $\alpha 16 / \mathrm{NOD}$, and BDC2.5/NOD mice were bred in the Joslin Diabetes Center barrier facility or were purchased from the Jackson Laboratory. Mice were monitored for diabetes as described previ- 
ously (21), and mice with serum glucose greater that $350 \mathrm{mg} / \mathrm{dl}$ were considered diabetic. For some experiments, diabetes was provoked in 6to 8-week-old female BDC2.5/NOD mice by intraperitoneal injection of CPA $(200 \mathrm{mg} / \mathrm{kg})$ using established protocols (23). To allow survival of $\mathrm{BDC} 2.5 / \mathrm{NOD}$ mice for analysis 35 days after CPA treatment, exogenous insulin was provided by subcutaneous insulin-releasing implants (LinBit; LinShin Canada Inc.). Animals were cared for in accordance with ethical guidelines of Joslin Diabetes Center and Harvard Medical School, and all studies were approved by the Joslin Diabetes Center Institutional Animal Care and Use Committee (\#99-20).

Histology. Pancreata were collected and fixed in 10\% neutral-buffered formalin (Sigma-Aldrich). Paraffin-embedded sections were stained with $\mathrm{H} \& \mathrm{E}$ and were examined for insulitis.

Anti-CD3 $m A$ b therapy. Anti-CD3 mAbs were purified from the supernatant of 145-2C11 hamster B cell hybridoma (ATCC) grown under the recommended conditions. $\mathrm{F}\left(\mathrm{ab}^{\prime}\right)_{2}$ fragments were generated [ImmunoPure $\mathrm{F}\left(\mathrm{ab}^{\prime}\right)_{2}$ Preparation Kit; Pierce Biotechnology Inc.] according to the manufacturer's protocol, and purity was confirmed by SDS-PAGE gel electrophoresis. Anti-CD3 mAb therapy was commenced within 1 week of NOD mice developing spontaneous diabetes. Mice with new-onset diabetes received an intravenous injection of $50 \mu \mathrm{g} 145-2 \mathrm{C} 11 \mathrm{~F}\left(\mathrm{ab}^{\prime}\right)_{2}$ fragments per day for 5 consecutive days (28). Mice were considered to have responded to therapy if blood glucose levels fell to the normal range and glycosuria resolved, and these benefits were maintained for at least 2 weeks. $\mathrm{F}\left(\mathrm{ab}^{\prime}\right)_{2}$ fragments prepared from hamster monoclonal antibodies against DNP-derivatized keyhole limpet hemocyanin (UC8-1B9; ATCC) were used for control studies.

MRI. A 4.7 Tesla microimaging system (PharmaScan; Bruker Biospin) was used to perform MRI. Mice were anesthetized by inhalation of isoflurane and were placed in a birdcage radio-frequency coil with an inner diameter of $36 \mathrm{~mm}$. The imaging protocol included multiple-slice and multiple-echo spin-echo sequences ( $\operatorname{Tr}, 2000 \mathrm{msec}$; TE, 6.5-195 msec; FOV, $3.5 \mathrm{~cm}$, matrix size, $128 \times 128$; slice thickness, $0.6 \mathrm{~mm}$ interleaved; number of excitations, 4). A total of 16 sequential coronal images were obtained to cover the entire pancreatic region.

Model MNPs (in this case, monocrystalline iron oxide nanoparticles [MION-47]) were injected at $20 \mathrm{mg} / \mathrm{kg}$ Fe to allow quantification of microvascular changes associated with pancreatic inflammation (15). MION-47 (Center for Molecular Imaging Research, Massachusetts General Hospital, Harvard Medical School) had a size of $22 \mathrm{~nm}$ and an R2 relaxivity of 69 $\mathrm{mMsec}^{-1}$ at $37^{\circ} \mathrm{C}(0.47 \mathrm{~T})$. Mice underwent scanning immediately before and after MION-47 injection, and a third scan was performed 24 hours after MION-47 administration to quantify microvascular leakiness.

Image analysis was performed using software custom built at the Center for Molecular Imaging Research and developed in Interactive Data Language (Research Systems Inc.). ROIs for analysis were defined manually on the pancreas or paraspinal muscles on 3 consecutive slices. To ensure that there were no volume-averaging effects with adjacent organs on calculated $\mathrm{T} 2$ values, the ROIs were propagated to adjacent slices and were modified as needed such that the windows on original and adjacent slices contained only the tissue of interest. T2 values for individual organs were calculated by fitting a standard exponential relaxation model to the data averaged over the entire ROI on each slice. Values less than background were not included in analysis (Figure 1). The mean T2 value for the 3 consecutive slices was then calculated to determine the value for each organ. For some experiments VVF and vascular leak were calculated as described previously (VVF, $\left[\ln \left(\mathrm{T} 2_{\text {before MNP }} /\right.\right.$ $\left.\left.\mathrm{T} 2_{\text {immediately after MNP }}\right)\right]$; vascular leak, $\left[\ln \left(\mathrm{T} 2_{\text {before }} \mathrm{MNP} / \mathrm{T} 2_{24 \mathrm{~h} \text { after MNP }}\right)(15)\right.$.

Statistical analyses. Data were analyzed using Student's $t$ test, assuming 2-tailed distribution, or Dunnett's multiple comparison test (GraphPad Prism 4 software; GraphPad Software Inc.). $P \leq 0.05$ was considered statistically significant.

\section{Acknowledgments}

We thank E. Hyatt for animal husbandry and N. Asinovski for tissue culture support. This work was funded by grants from the NIH (to D. Mathis, R. Weissleder, C. Benoist, and U. Mahmood) and by NIH grant R24 CA92782 (to R. Weissleder).

Received for publication March 15, 2005, and accepted in revised form June 7, 2005.

Address correspondence to: Diane Mathis, Joslin Diabetes Center, 1 Joslin Place, Boston, Massachusetts 02215, USA. Phone: (617) 264-2745; Fax: (617) 264-2744; E-mail: dm@joslin.harvard.edu.

Stuart E. Turvey's present address is: British Columbia Children's Hospital and University of British Columbia, Division of Infectious and Immunological Diseases, Vancouver, British Columbia, Canada.

Maria C. Denis's present address is: Biomedical Sciences Research Center, “Alexander Fleming” Institute of Immunology, Vari, Greece.
1. Bach, J.F. 1994. Insulin-dependent diabetes mellitus as an autoimmune disease. Endocr. Rev. 15:516-542.

2. Atkinson, M.A., and Eisenbarth, G.S. 2001. Type 1 diabetes: new perspectives on disease pathogenesis and treatment [review]. Lancet. 358:221-229.

3. Tisch, R., and McDevitt, H. 1996. Insulin-dependent diabetes mellitus. Cell. 85:291-297.

4. Imagawa, A., et al. 2001. Pancreatic biopsy as a procedure for detecting in situ autoimmune phenomena in type 1 diabetes: close correlation between serological markers and histological evidence of cellular autoimmunity. Diabetes. 50:1269-1273.

5. Diabetes Prevention Trial-Type 1 Diabetes Study Group. 2002. Effects of insulin in relatives of patients with type 1 diabetes mellitus. N. Engl. J. Med. 346:1685-1691.

6. Papaccio, G. 1993. Insulitis and islet microvasculature in type 1 diabetes. Histol. Histopathol. 8:751-759

7. Papaccio, G., Latronico, M.V., Pisanti, F.A., Federlin, K., and Linn, T. 1998. Adhesion molecules and microvascular changes in the nonobese dia- betic (NOD) mouse pancreas. An NO-inhibitor (L-NAME) is unable to block adhesion inflammation-induced activation. Autoimmunity. 27:65-77.

8. Carlsson, P.O., Sandler, S., and Jansson, L. 1998. Pancreatic islet blood perfusion in the nonobese diabetic mouse: diabetes-prone female mice exhibit a higher blood flow compared with male mice in the prediabetic phase. Endocrinology. 139:3534-3541.

9. De Paepe, M.E., Corriveau, M., Tannous, W.N., Seemayer, T.A., and Colle, E. 1992. Increased vascular permeability in pancreas of diabetic rats: detection with high resolution protein A-gold cytochemistry. Diabetologia. 35:1118-1124.

10. Sandler, S., and Jansson, L. 1985. Vascular permeability of pancreatic islets after administration of streptozotocin. Virchows Arch. A. Pathol. Anat. Histopathol. 407:359-367.

11. Jansson, L., and Sandler, S. 1986. Alloxan-induced diabetes in the mouse: time course of pancreatic $B$-cell destruction as reflected in an increased islet vascular permeability. Virchows Arch. A. Pathol. Anat. Histopathol. 410:17-21.
12. Majno, G., et al. 1987. A pancreatic venular defect in the BB/Wor rat. Am. J. Pathol. 128:210-215.

13. Kitagawa, Y., Desemone, J., and Mordes, J.P. 1993. Pancreas-specific venular labeling by monastral blue $\mathrm{B}$ in the $\mathrm{BB}$ rat: modulation by prostaglandins and their inhibitors. Immunopharmacology. 25:229-238.

14. McDonald, D.M., and Choyke, P.L. 2003. Imaging of angiogenesis: from microscope to clinic [review]. Nat. Med. 9:713-725.

15. Bremer, C., et al. 2003. Steady-state blood volume measurements in experimental tumors with different angiogenic burdens a study in mice. Radiology. 226:214-220.

16. Weissleder, R., et al. 2000. In vivo magnetic resonance imaging of transgene expression. Nat. Med. 6:351-355.

17. Denis, M.C., Mahmood, U., Benoist, C., Mathis, D., and Weissleder, R. 2004. Imaging inflammation of the pancreatic islets in type 1 diabetes. Proc. Natl. Acad. Sci. U. S. A. 101:12634-12639.

18. Harisinghani, M.G., et al. 2003. Noninvasive detection of clinically occult lymph-node metastases in 
prostate cancer. N. Engl. J. Med. 348:2491-2499.

19. Haskins, K., Portas, M., Bradley, B., Wegmann, D., and Lafferty, K. 1988. T-lymphocyte clone specific for pancreatic islet antigen. Diabetes. 37:1444-1448

20. Haskins, K., Portas, M., Bergman, B., Lafferty, K., and Bradley, B. 1989. Pancreatic islet-specific Tcell clones from nonobese diabetic mice. Proc. Natl. Acad. Sci. U. S. A. 86:8000-8004.

21. Katz, J.D., Wang, B., Haskins, K., Benoist, C., and Mathis, D. 1993. Following a diabetogenic $\mathrm{T}$ cell from genesis through pathogenesis. Cell. 74:1089-1100

22. Gonzalez, A., Andre-Schmutz, I., Carnaud, C., Mathis, D., and Benoist, C. 2001. Damage control, rather than unresponsiveness, effected by protective DX5 + T cells in autoimmune diabetes. Nat. Immunol. 2:1117-1125.

23. Andre-Schmutz, I., Hindelang, C., Benoist, C., and Mathis, D. 1999. Cellular and molecular changes accompanying the progression from insulitis to diabetes. Eur. J. Immunol. 29:245-255.

24. Pober, J.S., and Cotran, R.S. 1990. The role of endothelial cells in inflammation. Transplantation. 50:537-544.

25. Bohme, J., Schuhbaur, B., Kanagawa, O., Benoist, C., and Mathis, D. 1990. MHC-linked protection from diabetes dissociated from clonal deletion of
T cells. Science. 249:293-295.

26. Eisenbarth, G.S., et al. 2002. Insulin autoimmunity: prediction/precipitation/prevention type $1 \mathrm{~A}$ diabetes [review]. Autoimmun. Rev. 1:139-145.

27. Chatenoud, L., Thervet, E., Primo, J., and Bach, J.F. 1994. Anti-CD3 antibody induces long-term remission of overt autoimmunity in nonobese diabetic mice. Proc. Natl. Acad. Sci. U. S. A. 91:123-127.

28. Chatenoud, L., Primo, J., and Bach, J.F. 1997. CD3 antibody-induced dominant self tolerance in overtly diabetic NOD mice. J. Immunol. 158:2947-2954.

29. Belghith, M., et al. 2003. TGF-beta-dependent mechanisms mediate restoration of self-tolerance induced by antibodies to CD3 in overt autoimmune diabetes. Nat. Med. 9:1202-1208.

30. Bach, J.F., and Chatenoud, L. 2001. Tolerance to islet autoantigens in type 1 diabetes. Annu. Rev. Immunol. 19:131-161.

31. Herold, K.C., et al. 2002. Anti-CD3 monoclonal antibody in new-onset type 1 diabetes mellitus. N. Engl.J. Med. 346:1692-1698.

32. Signore, A., et al. 1999. In vivo imaging of insulitis in autoimmune diabetes. J. Endocrinol. Invest. 22:151-158.

33. Moore, A., Bonner-Weir, S., and Weissleder, R. 2001. Noninvasive in vivo measurement of betacell mass in mouse model of diabetes. Diabetes. 50:2231-2236.
34. Moore, A., Grimm, J., Han, B., and Santamaria, P. 2004. Tracking the recruitment of diabetogenic CD8+ T-cells to the pancreas in real time. Diabetes. 53:1459-1466.

35. Tree, T.I., and Peakman, M. 2004. Autoreactive T cells in human type 1 diabetes [review]. Endocrinol. Metab. Clin. North Am. 33:113-133.

36. Panagiotopoulos, C., Trudeau, J.D., and Tan, R. 2004. T-cell epitopes in type 1 diabetes. Curr. Diab. Rep. 4:87-94.

37. Lieberman, S.M., and DiLorenzo, T.P. 2003. A comprehensive guide to antibody and T-cell responses in type 1 diabetes. Tissue Antigens. 62:359-377.

38. Jaeger, C., et al. 1997. Persistent GAD 65 antibodies in longstanding IDDM are not associated with residual beta-cell function, neuropathy or HLA-DR status. Horm. Metab. Res. 29:510-515.

39. Bougneres, P.F., et al. 1988. Factors associated with early remission of type I diabetes in children treated with cyclosporine. N. Engl. J. Med. 318:663-670.

40. Palmer, J.P., et al. 2004. C-peptide is the appropriate outcome measure for type 1 diabetes clinical trials to preserve beta-cell function: report of an ADA workshop, 21-22 October 2001. Diabetes. 53:250-264.

41. Chatenoud, L. 2003. CD3-specific antibodyinduced active tolerance: from bench to bedside [review]. Nat. Rev. Immunol. 3:123-132. 